\title{
Experimental Determination of Crack Driving Forces in Integrated Structures
}

\author{
Jun $\mathrm{He}^{1}$, Guanghai $\mathrm{Xu}^{1}$, and Z. Suo ${ }^{2}$ \\ ${ }^{1}$ Intel Corporation, 5200 NE Elam Young Parkway, Hillsboro, Oregon 97124 \\ ${ }^{2}$ Division of Engineering and Applied Sciences, Harvard University, Cambridge, MA 02138
}

\section{ABSTRACT.}

For a crack in a brittle material in an integrated structure, the driving force $G$ is the reduction of the elastic energy in the structure, associated with the crack extending per unit area. In principle, $G$ can be calculated by solving a boundary value problem. In practice, however, such a calculation is prohibitively difficult for integrated structures of complex architectures, diverse materials and small features. The calculated value of $G$ is suspect when deformation properties and residual stress fields are poorly characterized. On the other hand, it costs little to make many replicates of an integrated structure, so that massive full-structure testing is affordable. We describe an experimental method to determine $G$. A crack, assisted by molecules in the environment (e.g., moisture), often extends at a velocity $V$ increasing with the crack driving force $G$. The $V$ $G$ function is specific to a given material and its environment. Once determined, the same function applies when this material is integrated in a structure with other materials, provided environmental molecules reach the crack front. In the integrated structure, an observed crack velocity, together with the known $V$ - $G$ function, provides a reading of the crack driving force. The observed crack velocity can be used to measure deformation properties of ultrathin films.

\section{INTRODUCTION}

Organosilicate glasses (OSGs), also known as carbon-doped oxides (CDOs), have recently been selected as low-permittivity dielectrics to replace silicon dioxide in on-chip interconnect structures (Maex [1], Hussein [2]). The OSGs are brittle. During fabrication and operation, an integrated structure acquires stresses from material deposition, thermal expansion, chemicalmechanical polishing, electromigration, etc. (Nix, [3], Suo [4], Maidenberg [5]). The stresses may cause the OSGs to grow cracks; Fig. 1 shows an example. Because the mechanical behavior of OSGs is similar to many candidate materials for future device structures, it is urgent to devise a protocol to prevent integrated structures from OSG cracking. For a crack in a given structure, the crack driving force, $\mathrm{G}$, is the reduction of the elastic energy in the structure associated with the crack extending per unit area, when the external mechanical load is rigidly held and does no work (Lawn [6]). An existing protocol is to calculate $G$ by solving a boundary value problem. Such solutions are accumulating for thin film structures $(\mathrm{Hu}$ [7], Hutchinson [8], Beuth [9] and Xia [10]). To prevent a crack from growing, the engineer must ensure that $G$ is below a threshold value. The latter is estimated from experimental measurements (Lawn [6]).

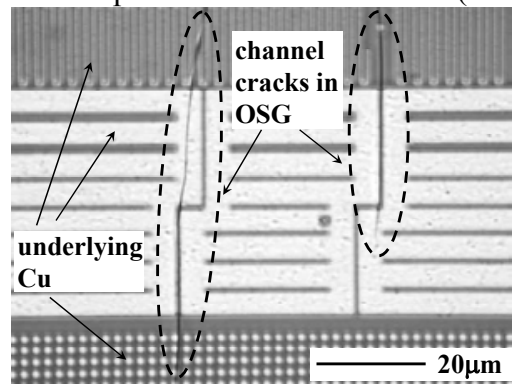

Figure 1. A plan view of a test structure shows two cracks running in OSG film on top of $\mathrm{Cu}$ interconnects 
The calculation of $G$ is prohibitively difficult for three-dimensional structures that integrate diverse materials. This is particularly so when the stress-strain relations of the constitute materials, as well as the residual stress fields in the structures, are poorly characterized. On the other hand, compared to large structures such as airplanes and ships, on-chip interconnects are inexpensive, making massive full-structure testing practical. These circumstances have motivated us to develop a method to measure crack driving force experimentally.

Our method relies on a familiar phenomenon: moisture-assisted crack growth. Water (and some other molecules) in the environment may participate in the process of breaking atomic bonds along the crack front (Lawn [6]). For the environmental molecules to reach the crack front and to break atomic bonds there, the crack extends at a velocity much below the sound speed in the material. The crack velocity $V$ is an increasing function of the crack driving force $G$. In recent years, such $V$ - $G$ functions have been measured for various dielectric films (Ma [11-12], Xu [13] and Cook [14]). Crack velocity as low as $10^{-12} \mathbf{m} / \mathbf{s}$ has been measured (Guyer [15]).

The $V-G$ function is specific to a given material and its environment. Once determined, the same function applies when this material is integrated with other materials in a structure, provided the environmental molecules reach the crack front. In such an integrated structure, an observed crack velocity, with the known $V$ - $G$ function, provides a reading of the crack driving force.

\section{CALIBRATING THE $V$ - $G$ FUNCTION}

Following Ma et al. [12], we measure the $V$ - $G$ function by observing the growth of a channel crack in an OSG film on a silicon substrate, subject to four-point bend (Fig. 2). Measure film residual stress, $\sigma_{R}$, by using the wafer curvature method (Nix [4]). Introduce initial flaws and then apply the stress in the film using a four-point fixture to bend the sample. Channel cracks emanate from the flaws. Measure the crack velocities using a digital camera. All tests reported in this work are conducted in an environmental chamber, at room temperature, with relative humidity of $30 \%$. Figure 3 shows two representative micrographs.

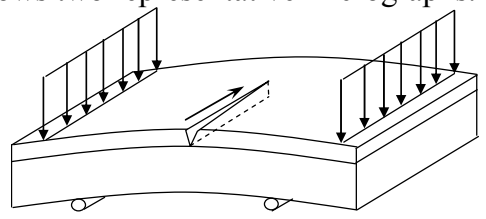

FIGURE 2. A schematic of the experimental setup. An OSG thin film is deposited on a silicon substrate. When the substrate is bent in a four-point bend fixture, a channel crack grows in the film. Measure the crack velocity as a function of the bending load. The data calibrate the $V-G$ function of the OSG.
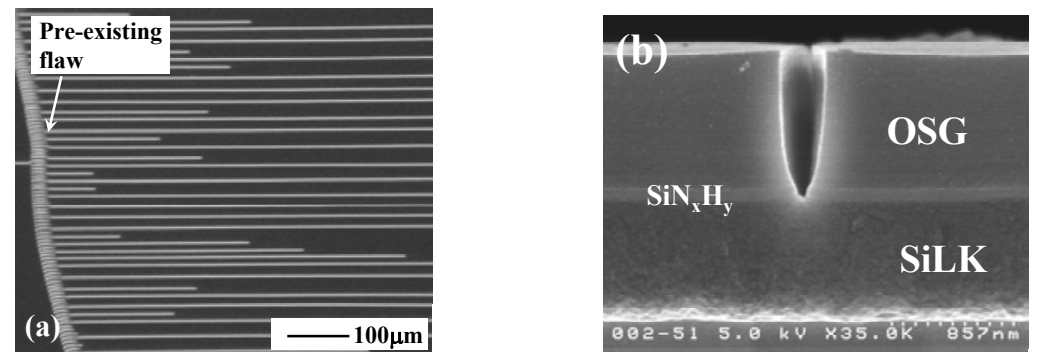

FIGURE 3. (a) An array of channel cracks grow in a $2250 \mathrm{~nm}$ OSG film on a silicon substrate. The cracks initiate at different times from the initial flaws. (b) On a silicon substrate, a film stack comprises a $834 \mathrm{~nm}$ SiLK (a spin-on organic film), a $60 \mathrm{~nm} \mathrm{SiN}_{\mathrm{x}} \mathrm{H}_{\mathrm{y}}$, and a $1061 \mathrm{~nm} \mathrm{OSG}$. 
The level of the bending load is measured by the radius of curvature of the substrate caused by the load. The net stress in the film, $\sigma$, is the sum of the residual stress and the bending stressing: $\sigma=\sigma_{R}+\sigma_{B}$. A dimensional consideration dictates that the crack driving force take the form

$$
\boldsymbol{G}=\boldsymbol{Z} \sigma^{2} \boldsymbol{h} / \overline{\boldsymbol{E}}
$$

where $h$ is the film thickness, and $Z$ is a dimensionless number. For an isolated channel crack in a thin film on a thick substrate, the number $Z$ varies with the elastic properties of the film and substrate, and has been calculated by solving the boundary value problem (Beuth [9]). We take Young's modulus and Poisson's ratio for silicon to be $165 \mathrm{GPa}$ and 0.28 , and those for the OSG to be $8.33 \mathrm{GPa}$ and 0.25 . From Beuth [9], we find that $\boldsymbol{Z}=1.096$.

Figure 4 gives the experimentally measured crack velocity $V$ as a function of the crack driving force $G$. Within the range of the measurement, our data fit an empirical relation:

$$
V / V_{*}=\exp \left(G / G_{*}\right)
$$

The fitting parameters are $\boldsymbol{V}_{*}=7.52 \times 10^{-16} \mathbf{m} / \mathbf{s}$ and $\boldsymbol{G}_{*}=0.106 \mathrm{~J} / \mathrm{m}^{2}$. A small change in the crack driving force causes a large change in the crack velocity. Our experimental setup can readily measure crack velocity between $10^{-9}$ to $10^{-4} \mathbf{m} / \mathbf{s}$. The $V-G$ function is sensitive to the environment. The crack velocity depends on humidity for a crack in ambient, and on the concentration of hydroxyl ions and hydrogen peroxide for a crack in an aqueous solution (Cook [14] and Guyer [15]). Consequently, within the measurable velocity range, we can access a wide range of $G$ by adjusting the environment. This preliminary account, however, does not pursue such an option.

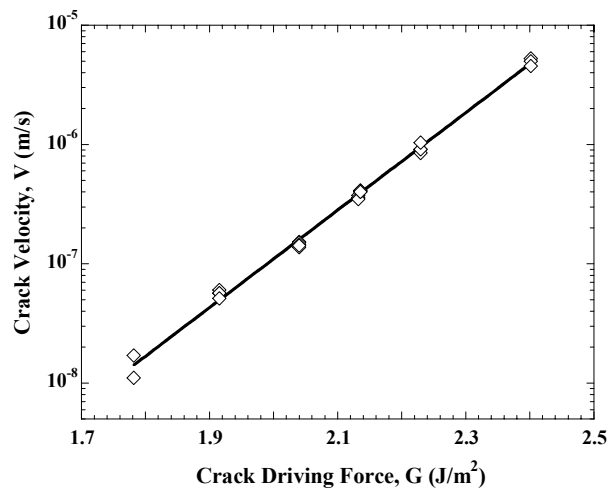

FIGURE 4. The $V$ - $G$ function measured by observing isolated channel cracks in an OSG film on Si substrate.

\section{READING THE CRACK DRIVING FORCE}

For a crack in the OSG in an integrated structure, an observed crack velocity, together with the $V$ $G$ functions in Fig. 4, provides a reading of the crack driving force. The method is accurate because a small variation in $G$ causes to a large variation in $V$. The method can readily measure the driving force $G$ of a crack in a complex structure such as that in Fig. 1. To ascertain the accuracy of the measurement, we use the multiple cracks in Fig. 3a as a demonstration of the method. In this case, the complexity arises from the multiple cracks, for which the mechanics is established. The measured velocity of isolated cracks as a function of the bending load calibrates $V-G$ function, as given in Fig. 4. At a fixed bending load, cracks initiate at different times. A new crack grows in a film stripe between two old cracks, whose fronts are much farther ahead. 
Because the old cracks partially relieve the stress in the stripe, the driving force for the new crack is lower than that for the old cracks. Consequently, the crack velocity decreases with the crack spacing. When the velocity of a new crack is measured, the $V$ - $G$ function in Fig. 4 provides a reading of $G$ for this crack.

Table 1. Experimental values of film stress, crack spacing, and crack velocity plotted in Fig.6

\begin{tabular}{|c|c|c|c|c|c|c|c|c|c|c|c|c|c|c|c|c|}
\hline Crack & $\mathrm{A}$ & $\mathrm{B}$ & $\mathrm{C}$ & $\mathrm{D}$ & $\mathrm{E}$ & $\mathrm{F}$ & $\mathrm{G}$ & $\mathrm{H}$ & $\mathrm{I}$ & $\mathrm{J}$ & $\mathrm{K}$ & $\mathrm{L}$ & $\mathrm{M}$ & $\mathrm{N}$ & $\mathrm{O}$ & $\mathrm{P}$ \\
\hline$\sigma(\mathrm{MPa})$ & 96.6 & 93.0 & 96.7 & 96.7 & 96.7 & 93.0 & 96.5 & 96.5 & 93.0 & 93.0 & 96.5 & 93.0 & 93.0 & 93.0 & 93.0 & 93.0 \\
\hline$S_{I}(\mu \mathrm{m})$ & 7.8 & 6.0 & 7.5 & 7.5 & 7.7 & 8 & 7 & $7 . .5$ & 8.5 & 10.5 & 8.0 & 11 & 20 & 16 & 20 & 20 \\
\hline$S_{2}(\mu \mathrm{m})$ & 5.0 & 8.0 & 5.8 & 6.0 & 5.7 & 7.8 & 6.7 & 7.5 & 8.8 & 10.5 & 8.0 & 11.5 & 11.3 & 17 & 18 & 20 \\
\hline$V(\mu \mathrm{m} / \mathrm{s})$ & 0.010 & 0.013 & 0.035 & 0.036 & 0.043 & 0.061 & 0.084 & 0.196 & 0.256 & 0.625 & 0.943 & 1.67 & 2.86 & 3.85 & 3.85 & 4.17 \\
\hline
\end{tabular}
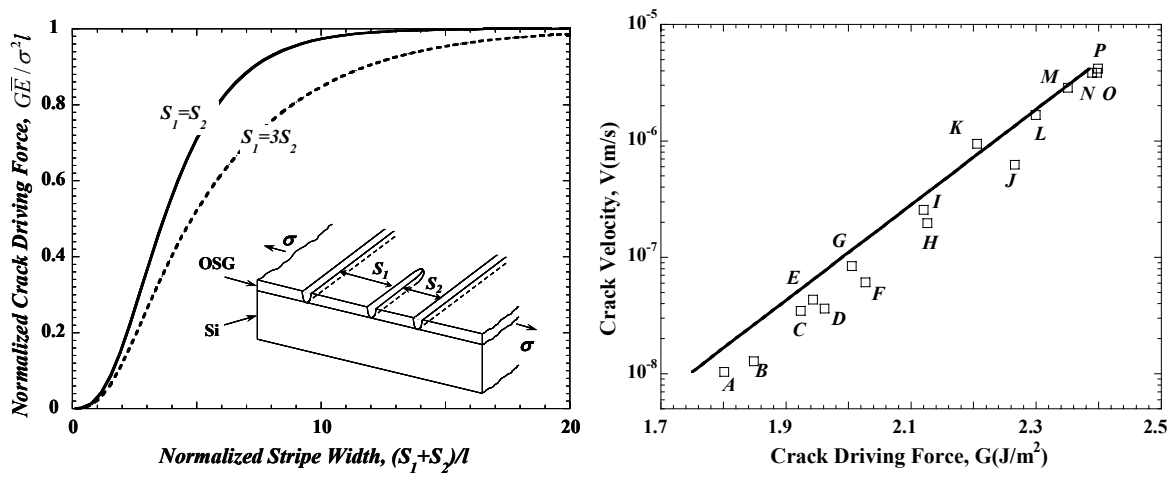

FIGUER 5. (a) The normalized crack driving force as a function of the normalized stripe width, as calculated from a shear lag model; (b) The solid line is the $V$ - $G$ function established by isolated cracks in the OSG film. Each data point corresponds to one of the 16 cracks in a film stripe, plotting the observed crack velocity against the crack driving force calculated from Eq. (4), using the observed crack spacings, as well as the stress, thickness and modulus of the OSG film.

To ascertain the accuracy of $G$ so measured, following Xia and Hutchisnon [8], we calculate $G$ for a crack extending between two old cracks. Consider the film stripe, of width $S$, between the two cracks. Near either crack, the stress in the stripe is partially relived. According to a shear lag model, the elastic energy stored per unit length of the stripe and the substrate underneath is

$$
U(S)=w S h-\frac{\sigma^{2} l h}{\bar{E}} \tanh \left(\frac{S}{2 l}\right),
$$

where $w$ is the elastic energy density, and $\sigma$ the net film stress, in the uncracked blanket film. The length $l$ scales with the width of the zone in which the stress is significantly relieved.

Next consider a new crack growing in the stripe, at distances $\boldsymbol{S}_{1}$ and $\boldsymbol{S}_{2}$ from the two old cracks (Fig. 5a). Far ahead the new crack front, the elastic energy per unit length is $\boldsymbol{U}\left(\boldsymbol{S}_{1}+\boldsymbol{S}_{2}\right)$. Far behind, the new crack splits the stripe into two, and the elastic energy per unit length is $\boldsymbol{U}\left(\boldsymbol{S}_{1}\right)+\boldsymbol{U}\left(\boldsymbol{S}_{2}\right)$. By definition, the driving force for the new crack, $G$, is the reduction of elastic energy in the structure associated with the new crack extends per unit area: $\boldsymbol{G h}=\boldsymbol{U}\left(\boldsymbol{S}_{1}+\boldsymbol{S}_{2}\right)-\boldsymbol{U}\left(\boldsymbol{S}_{1}\right)-\boldsymbol{U}\left(\boldsymbol{S}_{2}\right)$. This gives

$$
G=\frac{\sigma^{2} l}{\bar{E}}\left[\tanh \left(\frac{S_{1}}{2 l}\right)+\tanh \left(\frac{S_{2}}{2 l}\right)-\tanh \left(\frac{S_{1}+S_{2}}{2 l}\right)\right] .
$$


Figure 5(a) plots the crack driving force as a function of the stripe width. The crack driving force increases with the stripe width, and approaches a plateau when the stripe width exceeds some multiple of the relaxation length. A crack along the centerline of the stripe has a higher driving force than that running near either edge of the stripe. For Eq. (4) to recover the driving force for an isolated crack, Eq. (1), following Xia and Hutchinson [8], we will set the relaxation length to be $\boldsymbol{l}=\boldsymbol{Z} \boldsymbol{h}$.

In the experiment, we follow 16 cracks in various film stripes, recording the $\boldsymbol{S}_{1}$ and $\boldsymbol{S}_{2}$, as well as the net film stress (Table 1). Such data are used to calculate the crack driving forces from Eq. (4). Figure 5(b) plots the crack driving force calculated this way against the observed crack velocity. The agreement with the $V-G$ function determined from the isolated cracks is excellent.

\section{MEASURING ELASTIC MODULUS AND YIELD STRENGTH}

In an integrated structure, the driving force for a crack in one material may depend on deformation properties of all constituent materials. Consequently, the measured crack velocity can provide a reading of deformation properties. For example, we can measure Young's modulus of a thin film by using two test configurations. First, measure the $V$ - $G$ function of a thin film by using a test configuration in which $G$ is independent of the elastic constants of the film. This independence is a common feature for any specimen consisting of a thin film sandwiched between two thick substrates (Ma [11], Guyer [15]). Then, separately measure the crack velocity for the same film in the channel crack configuration, Fig. 2. Using the known $V-G$ function, the measured channel crack velocity provides a reading for $G$. For a channel crack, $G$ does depend on Young's modulus of the film, Eq. (1). Young's modulus can thus be deduced.

An alternative is to estimate Young's modulus of the film by just using the channel crack velocity measurements alone. The relaxation length $l$ increases with the ratio of the elastic modulus of the film to that of the substrate. Consequently, the velocities of multiple channel cracks contain the information of Young's modulus of the film.

The observed crack velocity can also provide a reading for the yield strength. To illustrate the idea, consider the film stack shown in Fig. 3b, consisting of OSG, $\mathrm{SiN}_{\mathrm{x}} \mathrm{H}_{\mathrm{y}}$ and $\mathrm{SiLK}$ films on a silicon wafer. The SiLK is a plastically deformable organic (Martin [16]), with Young's modulus 3.1 GPa and yield strength about $50 \mathrm{MPa}$ measured by nano-indentation. Fig. 6(a) plots the measured crack velocity as a function of a loading parameter. The data are impressive when compared with those for the OSG film by itself on silicon substrate. Evidently, the introduction of the SiLK underlayer makes the OSG film more vulnerable to cracking.

The remarkable difference is caused by the low modulus and the low yield strength of the SiLK underlayer. Fig. 6(b) plots the normalized crack driving force, $\boldsymbol{G} \overline{\boldsymbol{E}} / \boldsymbol{h} \sigma^{2}$, determined from the crack velocities, as a function of the stress in the OSG film. Also plotted is the value of $\boldsymbol{G} \overline{\boldsymbol{E}} / \boldsymbol{h} \sigma^{2}$ calculated using the finite element method, assuming that SiLK is linearly elastic. This value $\boldsymbol{G} \overline{\boldsymbol{E}} / \boldsymbol{h} \sigma^{2}=1.53$, as compared to $Z=1.096$ for the OSG on silicon, shows the effect of an underlayer of a low elastic modulus on the crack driving force. As evident in Fig. 6(b), the elastic effect, however, does not account for the measured crack driving force. We attribute the difference to plastic deformation in the SiLK underlayer.

For a channel crack in an elastic film on an elastic and perfectly plastic underlayer, the driving force is given by $\mathrm{Hu}$ [7]

$$
\frac{\boldsymbol{G} \overline{\boldsymbol{E}}}{\sigma^{2} \boldsymbol{h}}=\boldsymbol{A}+\frac{\sigma}{\sqrt{3} \sigma_{Y}},
$$


where the constant $A$ accounts for the elastic deformation, and $\sigma_{Y}$ is the yield strength of the SiLK underlayer. From the slope of the data line in Fig. 6(b), we estimate that $\sigma_{Y}=23 \mathbf{M P a}$. From the magnitude of the data line, we estimate that $\sigma_{Y}=41.6$ to $46.7 \mathrm{MPa}$. Both estimates are somewhat lower than that reported in the literature as well as values obtained by nanoindentation. The discrepancy might be due to the residual stress in the SiLK underlayer, and to Eq. (5) used to analyze the data. The latter was derived from an approximate model. Also, we have neglected the $\mathrm{SiN}_{\mathrm{x}} \mathrm{H}_{\mathrm{y}}$ layer in our analysis. A comprehensive analysis of these effects will be reported elsewhere.
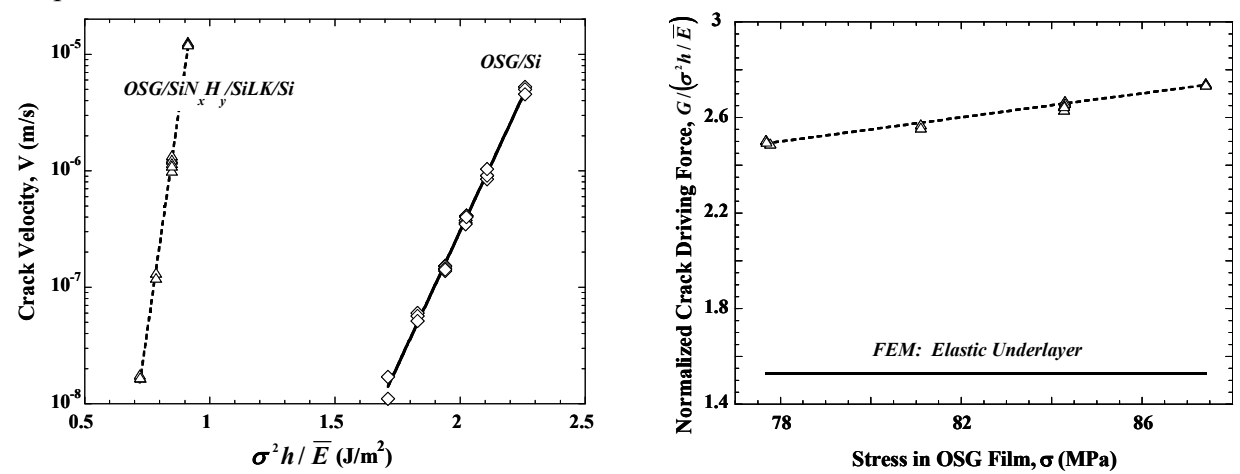

FIGURE 6. (a) The measured crack velocity as a function of a loading parameter. Two cases are compared: OSG/Si and $\mathrm{OSG} / \mathrm{SiN} / \mathrm{SiLK} / \mathrm{Si}$. The latter is more vulnerable to cracking. (b) The normalized crack driving force for an OSG film on a SiLK layer as a function of the stress in the OSG film. The data points are the readings from the observed crack velocity. The solid line in the lower part of the figure is calculated from the finite element method, assuming the SiLK underlayer is linearly elastic.

\section{REFERENCES}

1. K. Maex, M.R. Bakanov, D. Shamiryan, F. Iacopi, S.H. Brongersma and Z.S. Yanovitskaya, J. Appl. Phys. 93, 8793-8841 (2003).

2. M.A. Hussein and Jun He, IEEE Trans. Semicond. Manufact. In press (2004).

3. W.D. Nix. Metall. Trans. 20A, 2217-2245 (1989).

4. Z. Suo, pp. 265-324 in Volume 8: Interfacial and Nanoscale Failure (W. Gerberich, W. Yang, Editors), Comprehensive Structural Integrity (I. Milne, R.O. Ritchie, B. Karihaloo, Editors-in-Chief), Elsevier, Amsterdam, 2003.

5. D.A. Maidenberg, W. Volksen, R.D. Miller and R.H. Dauskardt. In press.

6. B. Lawn, Fracture of Brittle Solids. Cambridge University Press (1993).

7. M.S. Hu and A.G., Evans, Acta Metall. 37, 917-925 (1989).

8. J.W. Hutchinson and Z. Suo, Advances in Applied Mechanics 29, 63-161 (1991).

9. J.L. Beuth, Int. J. Solids Structures 29, 1657-1675 (1992).

10. Z.C. Xia and J.W. Hutchinson, J. Mech. Phys. Solids 48, 1107-1131 (2000).

11. Q. Ma, J. Mater. Res. 12, 840-845 (1997).

12. Q. Ma, J. Xie, S. Chao, S. El-Mansy, R. McFadden and H. Fujimoto, Mater. Res. Soc. Symp. Proc. 516, 331-336 (1998).

13. Guanghai Xu; Jun He, E. Andideh; J. Bielefeld and T. Scherban, Proc of the 2002 International Interconnect Technology Conference, pp 57-59, June 7-9, San Francisco, California (2002).

14. R.F. Cook and E.G. Liniger, J. Electochem. Soc. 146, 4439-4448 (1999).

15. E. P. Guyer and R. H. Dauskardt, submitted for publication.

16. S.J. Martin, J.P. Godschalx, M.E. Mills, E.O. Shaffer and P.H., Townsend. Advanced Materials 12, 1769-1778 (2000). 